\title{
Model-Free Functional MRI Analysis Using Kohonen Clustering Neural Network and Fuzzy $C$-Means
}

\author{
Kai-Hsiang Chuang, Ming-Jang Chiu, Chung-Chih Lin, Student Member, IEEE, and Jyh-Horng Chen,* Member, IEEE
}

\begin{abstract}
Conventional model-based or statistical analysis methods for functional MRI (fMRI) suffer from the limitation of the assumed paradigm and biased results. Temporal clustering methods, such as fuzzy clustering, can eliminate these problems but are difficult to find activation occupying a small area, sensitive to noise and initial values, and computationally demanding. To overcome these adversities, a cascade clustering method combining a Kohonen clustering network and fuzzy $c$ means is developed. Receiver operating characteristic (ROC) analysis is used to compare this method with correlation coefficient analysis and $t$ test on a series of testing phantoms. Results show that this method can efficiently and stably identify the actual functional response with typical signal change to noise ratio, from a small activation area occupying only $0.2 \%$ of head size, with phase delay, and from other noise sources such as head motion. With the ability of finding activities of small sizes stably, this method can not only identify the functional responses and the active regions more precisely, but also discriminate responses from different signal sources, such as large venous vessels or different types of activation patterns in human studies involving motor cortex activation. Even when the experimental paradigm is unknown in a blind test such that model-based methods are inapplicable, this method can identify the activation patterns and regions correctly.
\end{abstract}

Index Terms-Analysis, functional MRI, fuzzy clustering, Kohonen clustering network.

\section{INTRODUCTION}

$\mathbf{F}$ UNCTIONAL magnetic resonance imaging (fMRI) with high temporal and spatial resolution is a potential method to map rapid and fine activation patterns of the human brain [1]-[5]. According to both theoretical estimations and experimental results [1], [6], [7], activated signal variation is very low on a clinical scanner. Thus, analysis methods are required to find the response waveforms and the associated activated regions. Generally these methods can be divided into two

Manuscript received June 10, 1998; revised September 23, 1999. This work was supported in part by the National Science Council, Taiwan, R.O.C., under Grant NSC86-2314-B-002-307-M08. The Associate Editor responsible for coordinating the review of this paper and recommending its publication was X. Hu. Asterisk indicates corresponding author.

K.-H. Chuang is with Department of Electrical Engineering, National Taiwan University, Taipei, Taiwan, R.O.C.

M.-J. Chiu is with Department of Electrical Engineering, National Taiwan University, Taipei, Taiwan, R.O.C., and with the Department of Neurology, College of Medicine, National Taiwan University, Taipei, Taiwan, R.O.C.

C.-C. Lin and *J.-H. Chen are with Department of Electrical Engineering, National Taiwan University, Taipei, Taiwan, R.O.C.

Publisher Item Identifier S 0278-0062(99)10412-9. categories depending on whether they require prior knowledge about activation patterns: model based and model free.

In model-based approaches, brain mapping is formed either by correlating with the estimated functional response, such as a boxcar waveform, in the time or frequency domain [8], [9] or by comparing mean differences between the control and the activated states statistically [10], [11]. Since they provide some statistical inference and are easy to implement, these methods are most widely used. However, the actual functional response, which differs from subject to subject and from condition to condition even under simple visual or motor tasks, is far more complicated than the usually assumed boxcar waveform or more sophisticated response models [12], [13]. Analysis methods based on any simple response model will become less effective as experimental conditions become more complex or when applied to data of different subjects. This problem is crucial when analyzing single trial data, where the actual responses are complex and unknown and the exact period of activated state is hard to define [14], [15]. In fact, even when the exact temporal response is not so crucial in analysis, the activation map produced by a model-based method only reveals the areas with the activities we anticipated, i.e., it is a biased activation map rather than the actual one and the averaged functional response in such regions is also biased. This fallacy could lead to incorrect conclusions when investigating characteristics of functional response under different modalities, stimulating rates, stimulation intensities, etc.

To study dynamic properties of actual response, one strategy is to use the averaged waveform in a region of interest (ROI) as a reference for correlation. The other is to study the responses in some ROI's only [16]. The previous approach limits our scope to the region with activity resembling the one in the selected ROI. The latter limits our ability to find activation in previously unexpected regions. Besides, selecting ROI's from a large amount of data is very tedious. Despite the situation where actual response is crucial the presence of hemodynamic response delay is still a problem, especially when using some statistical test, such as the $t$ test, for analysis. Although discarding images possibly covering this transition can avoid the problem, this strategy overlooks the physiological information in the transient hemodynamic response.

To eliminate the bias and limitation of model-based analysis methods and to satisfy the demand to analyze data with 
complicated experimental conditions, analysis methods that do not rely on any assumed model of functional response are necessary. There are two kinds of model-free methods. One, such as principal component analysis (PCA) [17], [18] or independent component analysis (ICA) [19], [20], transforms original data into high-dimensional vector space to separate functional response and various noise sources from each other. The major problem of PCA is that it only diminishes second-order dependency between each component. ICA can reduce higher order dependency but it is still limited by stationary distribution and the linear mixture assumption [20]. In addition, both methods require much more memory and processing time than other methods and therefore can not provide real-time analysis.

The other method, such as the fuzzy clustering analysis (FCA) [21] or self-organizing map (SOM) [22], attempts to classify time signals of the brain into several patterns according to temporal similarity among these signals. In FCA, similarity is determined by a fuzzy membership function between each input waveform and cluster centroid [23]. With a proper selection of the fuzzy factor and cluster number, FCA is shown to be able to identify various patterns, such as artifact-related activities, venous contribution, different activation levels, etc., from the brain [24]-[26]. However, the result of FCA is highly dependent on the selection of the fuzzy factor and cluster number, especially when identifying features of small sizes. Since functional activation areas, compared to the brain are very small, this problem becomes crucial when finer activation structures are to be differentiated. If we tried to enhance the detectability by increasing the cluster number, the complexity of this algorithm will make the analysis very inefficient. Furthermore, FCA is sensitive to noise and initial values. This may make it either unable to converge or cause it converge to an erroneous result in some conditions [27]. Methods have been proposed to address some of these problems. For example, merging redundant clusters during iteration can improve processing speed [28]. Modifying the original Euclidean-based distance definition by correlation can reduce the sensitiveness to noise and initial values [27], but features of different signal change levels, which may have important physiological meaning [29], could be hard to separate using this strategy.

Kohonen's SOM [30], which attempts to reduce the dimension while preserving the topological structure of a data set, can also be used in clustering. Based on competitive learning, it is not as computer intensive as FCA. Thus, features of small sizes can be more efficiently identified using more clusters. The problem of this algorithm is that it is not based on optimization of any object function, hence, it does not ensure convergence to optimal results and still will be sensitive to noise level and initial value [31].

Using one-step processing to identify fine structures from a large noisy data set such as functional MR images is difficult for all clustering methods. In this paper, we propose a cascade classification scheme to overcome the problems: difficulty in finding activation of small area, sensitivity to noise and initial values, long processing time and large memory demand. A fast and robust clustering neural network, the Kohonen clustering

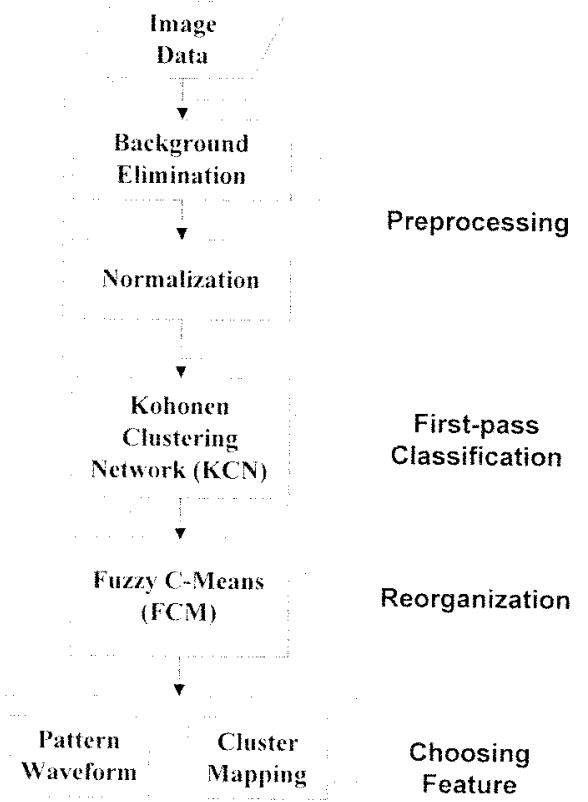

Fig. 1. Data flow of the model-free fMRI analysis method cascading Kohonen clustering neural network and FCM. See text for detailed description.

network $(\mathrm{KCN})$, is used in the first stage to classify fMRI time signals into many clusters to avoid misclassification. Then fuzzy $c$ means (FCM) is used to reorganize and merge the identified features into primary ones in the second stage. By combining both clustering methods, analysis power can be improved without sacrificing efficiency. Receiver operating characteristic (ROC) analysis [32], [33] was performed on a series of computer generated testing phantoms to evaluate the properties and to compare the performance of this method with two of the most popular model-based analysis methods, correlation coefficient analysis (CCA) [8] and student $t$ test [10]. Human studies with regular and arbitrary paradigm designs were conducted to evaluate its effectiveness under actual conditions. A blind test with unknown-paradigm data from a remote MR institute is also demonstrated. Under a moderate noise level, this method can efficiently and stably extract functional responses with different signal change levels, temporal delays, complex activation patterns, etc., from regions occupying only $0.2 \%$ brain area. With the ability to identify features of small sizes, activation can be localized more precisely. Other noise sources, such as head motion, or activation from different signal sources can be discriminated.

\section{MATERIALS AND METHODS}

\section{A. Analysis Method}

Analysis begins by eliminating undesired signals in the background and normalizing fMRI time signals (Fig. 1). Then, preprocessed time signals are clustered by two cascaded clustering methods. The purpose of the first stage clustering is to diminish the size of fMRI data to some extent, but not too much, to avoid misclassification of ambiguous features and features of small sizes. Thus, we need a fast and robust 
clustering method to handle the large amount of fMRI data. In this study, an optimization-based Kohonen clustering neural network is used. The purpose of the second clustering method is to reorganize and merge the similar features identified in the first stage into primary ones. Because FCM, based on the the fuzzy membership function, is good at separating similar and ambiguous features, it is used in the second stage. After finishing classification, all patterns and their associated regions are displayed for choosing patterns of interest.

1) Preprocessing: This stage is composed of background elimination and normalization:

a) Background elimination: To increase analysis efficiency, only signals in the brain are processed. Pixels with values lower than a certain threshold are regarded as background. Empirically, we set the threshold value to be $1 / 10$ of the highest intensity in the input image.

b) Normalization: Because we are only interested in the signal variation of functional MR images rather than their baseline signal intensities, the signal of each pixel will be subtracted by its mean value over the entire image sequence to eliminate the dependency of baseline intensity level. Not doing this will make the subsequent clustering procedures classify signals according to their baseline levels, leading to a result such as that of image segmentation. This is especially important when signals are acquired using surface coils.

2) Kohonen Clustering Network: Although the algorithm of SOM is not computer intensive, it is not guaranteed to converge to the optimal result due to its nonoptimization property. This property may also lead to erroneous classification under improper initial values or high noise levels. To reduce the sensitivity to noise and initial values, while preserving the fast computation property, an optimization-based modification proposed by Pal et al. [31] is used in this study.

The clustering procedure begins by selecting the desired cluster number $C_{K}$. After initializing $C_{K}$ cluster feature vectors, preprocessed time course signals are compared with each feature in the pixel-by-pixel manner to find their best matching feature, the winner. Each pixel is assigned to belong to its winner cluster. Euclidean distance is used to determine the similarity between input vector and cluster features. Then every cluster features, not just the winner, are modified according to their distance between the input vector. The nearer the cluster feature is to the input, the more it modifies itself toward that input vector. After matching and learning the characteristics of every input vector, this process iterates until cluster features do not change very much.

3) Fuzzy C-Means: Fuzzy clustering, different from hard clustering, does not make clear-cut classification of a data set. Instead, it only evaluates the probability to each cluster that the input belongs, just as a human does. So it is more suitable in classifying ambiguous data. Because many cluster features identified by $\mathrm{KCN}$ resemble each other, we use FCM [23] to reorganize and merge them into fewer features.

This clustering also begins by selecting the desired cluster number $C_{F}$ and randomly initializing $C_{F}$ cluster features. In our study, $C_{F}$ is roughly $1 / 5$ of $C_{K}$. The goal of FCM is to create a fuzzy membership matrix $\mu$ in which each element $\mu_{i j}$ describes the probability that the $j$ th input vector, $v_{j}$, belongs to the $i$ th cluster feature, $u_{i}$. To determine this membership matrix, the basic concept is that if the $j$ th input matches one cluster feature perfectly, the probability belonging to that cluster, $n$, should be one $\left(\mu_{n j}=1\right)$ and the probability belonging to other clusters should be zero $\left(\mu_{i j}=0\right.$ for $\left.i \neq n\right)$. If there is no perfectly matching cluster, the more an input vector resembles a cluster feature, the higher their membership is. The equation is of the form

$$
\mu_{i j}=\frac{\left(1 /\left\|\boldsymbol{v}_{j}-\boldsymbol{u}_{i}\right\|^{2}\right)^{1 / m-1}}{\sum_{k=1}^{C_{F}}\left(1 /\left\|\boldsymbol{v}_{j}-u_{k}\right\|^{2}\right)^{1 / m-1}}
$$

where $m$ is the fuzzy factor that determines the degree of fuzziness of the membership function. After finding the membership matrix, each cluster feature is updated according to all input vectors and the membership between them. This procedure will iterate until the membership function converges. Then we assign each input vector to the cluster with the highest probability between them or to the cluster with not only the highest probability but also above a threshold value.

4) Choosing Features: After the classification procedure is completed, one or more clusters will be selected to form the activation map. This can be done by manually or automatically comparing the temporal properties of the identified patterns. For new studies where the response of interest may not be estimated in advance, manual selection can be performed interactively using a GUI tool developed in our laboratory. Otherwise, clusters having high correlation coefficients $(C C)$ with respect to the paradigm are selected automatically.

\section{B. Phantom Design}

Since $\mathrm{KCN}$ is the core of information extraction in this analysis method, the result will largely depend upon it. To understand the performance and the characteristics of $\mathrm{KCN}$, five kinds of testing conditions were investigated using computergenerated phantoms. These conditions included noise level, activation area size, response phase delay, complex response, and head motion. Each phantom image consisted of several ellipses with different baseline intensities. Gaussian random noise was added in real and imaginary parts of Fourier domain to emulate actual noise distribution. The fMRI time series was composed of 36 images with an exponentially rising and falling functional activation waveform modulated by the testing condition added to a specific region. Two model-based methods, CCA and $t$ test, were also compared using the same phantoms. The boxcar reference waveform was used in CCA.

The usual functional signal change to noise ratio $(\Delta$ SNR) on conventional 1.5-T scanners could be as low as 1.5 or even lower. Therefore, phantoms with $\Delta$ SNR $=$ $1.0,1.25,1.5,1.75$, and 2.0, respectively, were studied. Examples of activation waveforms from two pixels with $\Delta$ SNR $=1.0$ and 2.0 are shown in Fig. 2 (A1) and (B1), respectively. The activation area sizes were fixed at $0.2 \%$ of head size in this condition.

Because the learning algorithms of $\mathrm{KCN}$ and FCM update cluster features by all input vectors iteratively, the more a type of input feature is presented the more probable this feature 

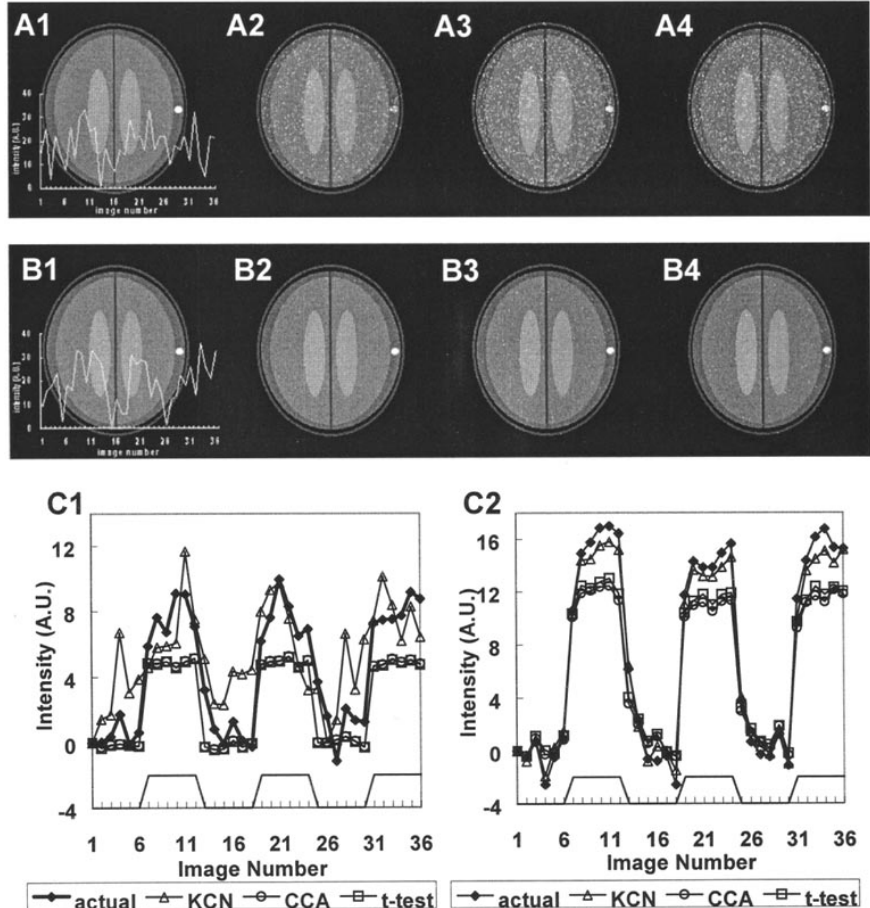

Fig. 2. (A) and (B) show the activation maps formed by the analysis methods under $\Delta \mathrm{SNR}=1.0$ and 2.0, respectively. The designed activation region and a sample of response waveform from a pixel in this region are shown in (A1) and (B1). The activation maps of $\mathrm{KCN}, \mathrm{CCA}$, and t-test are shown in (2), (3), and (4), respectively. (C1) and (C2) are the averaged waveforms in the activation regions identified by the three analysis methods under $\triangle \mathrm{SNR}=$ 1.0 and 2.0, respectively. It should be noted that although CCA and $t$ test performed better than KCN when $\triangle \mathrm{SNR}$ was 1.0, the averaged waveforms were biased toward the boxcar paradigm. When the noise level was lower, KCN surpassed both model-based methods and the averaged waveform was closer to the actual one.

will be identified. This means that a feature of a very small population could be concealed. This is very critical for fMRI analysis because the functioning area is typically very small compared with the whole brain. To test the ability to find features of a very small population, we designed six sets of phantoms with activation regions occupying $0.087 \%, 0.2 \%$, $0.33 \%, 0.5 \%, 0.67 \%$, and $0.83 \%$ of the designed head area. The SNR's were set at 2.0 and other noise levels were also studied for phantoms with larger activation area sizes.

In different sensory fMRI studies, phase lags of responses may be different for different subjects and processing systems involved. Since the underlying mechanism is not fully understood, this delay cannot be estimated under all conditions. Hence, the analysis power of model-based methods would be degraded while model-free methods, on the contrary, could be able to find the response pattern without being biased by any assumed model. To test the influences of delayed response, phantoms with phase delay between simulated response and the paradigm ranging from $\pi / 6, \pi / 3, \cdots$ to $\pi$ were inspected. Their activation areas were fixed at $0.2 \%$ of head size and SNR's were fixed at 2.0.

In addition to hemodynamic delay, the actual response pattern may be very complex under different stimulation or incorporated cortical region. We simulated the response under sustained visual stimulation [13] with fatigue- or adaptation- like response and poststimulus undershoot by an exponential function with a time constant of $2 \mathrm{~min}$. The $\Delta \mathrm{SNR}$ was set at 1.5 and the activation area was $0.2 \%$ of head size.

Gross head motion is one of the major artifacts in fMRI [34]. It will produce large signal variations on the edge of different parenchyma, especially between the cortex and cerebrospinal fluid (CSF), thus decreasing analysis power and leading to erroneous interpretation of analysis results. Since this kind of variation is different from other signal sources, model-free analysis methods could be able to identify this signal pattern. Effects of head motion on analysis methods were studied using a phantom with a sudden rotation from 0.1 to $0.5^{\circ}$ in the middle of the image sequence. Their activation areas were fixed at $0.2 \%$ of head size and $\Delta$ SNR's were 2.0.

\section{Evaluation-ROC Analysis}

To evaluate the properties of this method and to compare its performance with $\mathrm{CCA}$ and $t$ test, ROC analysis was performed. There are two parameters in ROC analysis: sensitivity and specificity. In our study, sensitivity is the proportion of the activation site identified correctly, and specificity is the proportion of the inactive region identified correctly. Conventionally, the trajectory of both parameters are plotted under different thresholds by taking 1 specificity, also known as the false positive fraction, as its $x$ axis and sensitivity, also known as the true positive fraction, as its $y$ axis to form an ROC curve. Since the ideal value of sensitivity and specificity is one, any curve corresponding to a certain method closest to the ideal upper left corner of a ROC plot will be the method of choice.

To plot the ROC curve for both model-based analysis methods we varied the threshold of CCA from $\mathrm{CC}=0.10$, $0.15, \cdots$, to 0.50 , and the threshold of $t$ test from $t$ value $=$ $0.5,1.0, \cdots$, to 4.0 . For our proposed method, the threshold was chosen to be the output cluster number. This was because more clusters, such as a higher threshold, tended to have a higher value of specificity and a lower value of sensitivity. The cluster number of $\mathrm{KCN}$ was varied from $5,10,15, \cdots$, to 80 . Larger cluster number was also studied in smaller activation area size condition. For FCM, the procedure is similar despite the fact that the cluster number only varied from $5,6, \cdots$, to 10 . The sensitivity and specificity of KCN or FCM under each cluster number were determined from the cluster with the highest correlation with respect to the paradigm. Then we compared the activation maps and the averaged waveforms in these regions, with all methods, under optimal threshold or classifying conditions, i.e., the conditions closest to the left upper corner of ROC curves.

\section{Performance Test}

In addition to the analysis power, two other important properties of our proposed method are computation time efficiency and insensitivity to initial values. The required computation time for a clustering algorithm to converge depends on many factors, such as the complexity of the algorithm itself, the error tolerance, the size of input data, the cluster number, the speed of the computer, etc. The processing time of CCA and $t$ test is 
very short due to the noniterative property of their algorithms. $\mathrm{KCN}$ and FCM, on the contrary, require much more time to converge. We compared the performance of CCA, $t$ test, $\mathrm{KCN}$, FCM, and our cascade method (KCN + FCM) using the same testing phantom. Since the cluster number will affect computation time, we also studied the converging time of $\mathrm{KCN}$ and FCM under different cluster numbers. All algorithms were written in $\mathrm{C}$ and compiled by a GNU C compiler (version 2.7.2, Free Software Foundation, MA, USA) with optimization running on a Sun SPARCstation 20 (Sun Microsystems, CA, USA). In addition, to test the sensitiveness to initial values, $\mathrm{KCN}$ was run ten times to evaluate the variation in sensitivity. Results under different $\Delta$ SNR were also studied.

\section{E. Human Experiment Design}

1) Regular Versus Arbitrary Paradigm Design: Motor cortex activation experiments were conducted using two kinds of paradigm designs. One was the traditional regular paradigm, which was composed of three repeated cycles of resting-stimulating states with 12 images in each state. During stimulating states, the subject was instructed to tap his or her fingers with self-paced complex finger movement patterns, such as 1-3-2-4-4-2-3-1 (1 represents the index finger, 2 the middle finger, and so on).

To test the ability to do blind analysis, we performed an arbitrary paradigm experiment on the same subject after the regular paradigm. In this experiment, a functional MR image series was divided into six blocks as in the regular paradigm experiment, but the stimulating blocks were selected voluntarily by the MRI operator. In addition, this paradigm was concealed from the person who analyzed the resulting data. The analysis method in this case did not have any given pattern to correlate with.

Four healthy subjects were investigated (three males and one female, ages 24-33). All experiments were performed on a standard 1.5-T GE Signa MR imager at National Taiwan University Hospital. Images were acquired using dual 3-in TMJ array coils positioned on the lateral sides of the parietal lobe by the desired motor cortex. Before each experiment, temporal stability was tested using a standard calibration phantom and the same fMRI imaging sequence stated below. If maximum temporal signal variation was below $1 \%$, fMRI experiments proceeded. Sagittal T1-weighted images were acquired to localize the desired slice. The oblique axial slice crossing the primary motor cortex was selected. The fMRI series were acquired using the standard FLASH pulse sequence with first-order flow compensation. Imaging parameters were $\mathrm{TR}=100 \mathrm{~ms}, \mathrm{TE}=40 \mathrm{~ms}$, flip angle $=20^{\circ}, \mathrm{FOV}=24 \times$ $24 \mathrm{~cm}$, matrix size $=256 \times 128$, slice thickness $=4 \mathrm{~mm}$, $\mathrm{NEX}=1$.

2) Blind Test: The power of model-free analysis is to identify actual functional response and other activity in the brain without any prior knowledge or assumption. To verify this, a fMRI data set from Kaohsiung Veterans General Hospital (KVGH), about $300 \mathrm{~km}$ away from our department, was analyzed without any knowledge about the actual paradigm. Then identified patterns and associated brain regions were

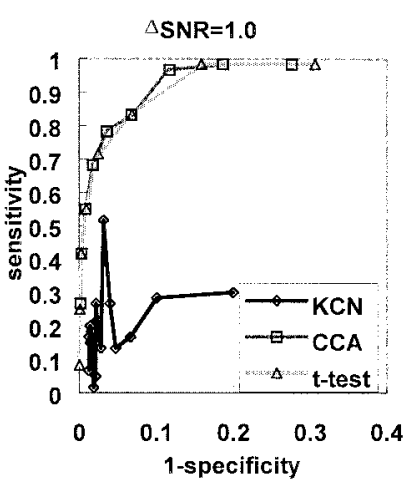

(a)

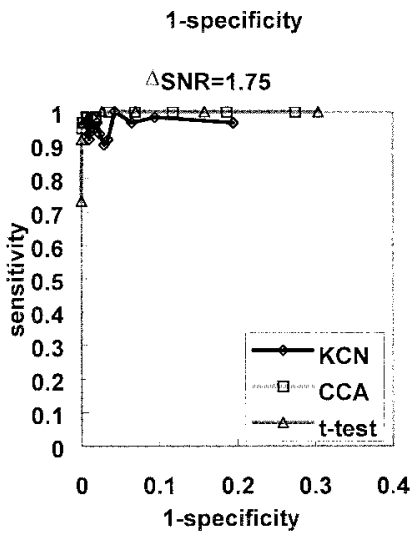

(c)

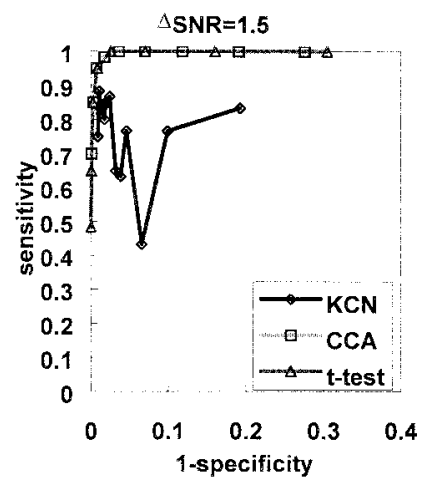

(b)

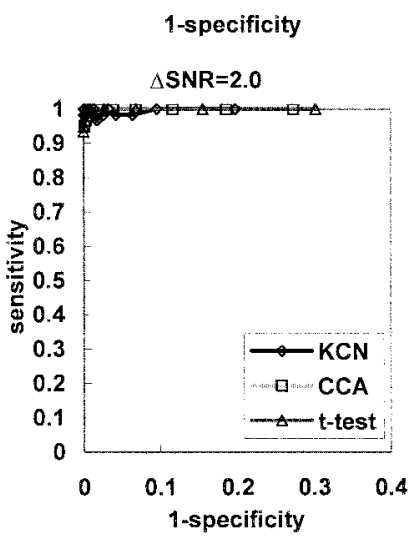

(d)
Fig. 3. ROC curves under $\Delta \mathrm{SNR}=$ (a) 1.0, (b) 1.5, (c) 1.75, and (d) 2.0. The performance of KCN was inferior to CCA and $t$ test when $\triangle$ SNR was very low. But it reached nearly the same level as both model-based methods when the SNR was higher than 1.75. Furthermore, it surpassed CCA and $t$ test as $\Delta$ SNR $=2.0$.

sent back for verification. The actual paradigm and imaging parameters were not provided until analysis was finished.

\section{RESULTS}

\section{A. Phantom Studies}

1) Dependence on Signal Change to Noise Ratio: Fig. 2 shows the activation maps and the averaged time-course waveform in the activation regions identified by $\mathrm{KCN}, \mathrm{CCA}$, and $t$ test under $\Delta$ SNR $=1.0$ [Fig. 2(A)] and 2.0 [Fig. 2(B)]. Under the noisiest condition $(\Delta \mathrm{SNR}=1.0)$, none of the methods could identify the actual activation region very well. Even though CCA and $t$ test, based on their assumed model, performed better than $\mathrm{KCN}$, their averaged responses were biased by the boxcar paradigm [Fig. 2(C1)]. At the higher $\Delta$ SNR condition, the activation pattern identified by $\mathrm{KCN}$ more resembled the designed response [Fig. 2(C2)] and the activation region is more precise. The ROC analysis (Fig. 3) showed that as $\Delta$ SNR increased, KCN with its sensitivity boosted quite quickly began to surpass both model-based methods. The performance of KCN became comparable to CCA and $t$ test when the $\Delta$ SNR was higher than 1.75.

In addition to the general trend, the relation between the $\Delta$ SNR and cluster number could be elucidated by plotting 


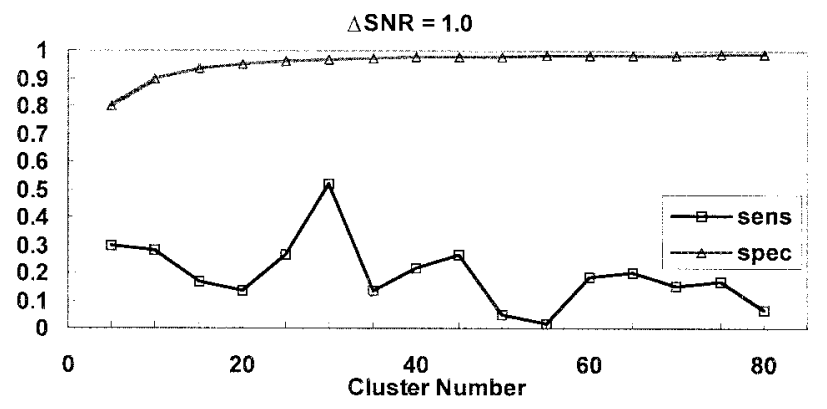

(a)

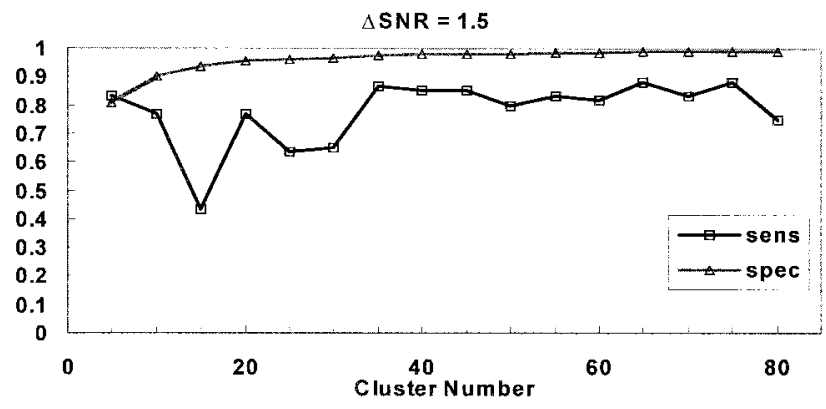

(b)

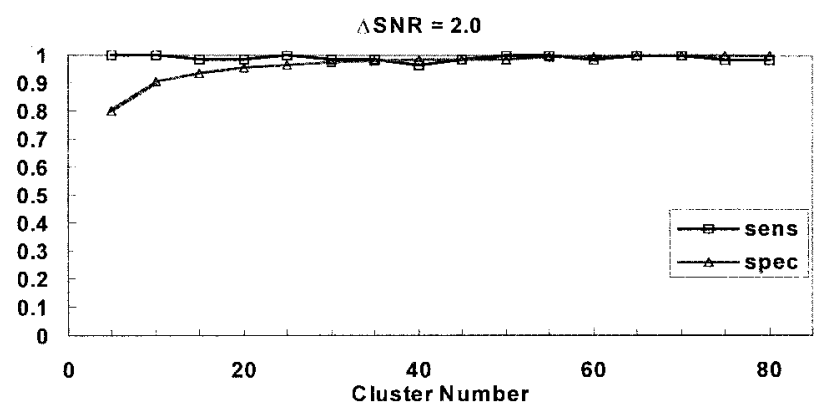

(c)

Fig. 4. Trends of sensitivity (the lines marked as sens) and specificity (marked as spec) with respect to cluster number under $\Delta \mathrm{SNR}=$ (a) 1.0, (b) 1.5, and (c) 2.0. Specificity increased monotonically with cluster number regardless of noise level. Sensitivity did not have a clear trend under the same noise level, but tended to oscillate randomly when the $\triangle$ SNR was low. Sensitivity became stable as $\Delta$ SNR increased. Thus $\Delta$ SNR was more influential on sensitivity than cluster number.

sensitivity and specificity versus cluster number (Fig. 4). In general, specificity increased with cluster number monotonically, regardless of the noise level. Sensitivity, however, did not have a clear trend with respect to cluster number but tended to oscillate randomly when the $\Delta$ SNR became lower. But for moderate noise levels, e.g., $\Delta$ SNR $>1.5$, sensitivity did not change very much with respect to cluster number.

2) Dependence on Activation Area Size: Since CCA and $t$ test analyze each pixel individually, they are not affected by the changes in activation area size (Table I). Under the smallest activation area size condition ( $0.087 \%$ of the head size), both model-based methods identified the actual activation region correctly with only a few misidentifications, as in the case of high $\Delta$ SNR. KCN also identified the actual activation region, but it misidentified more than both modelbased methods. However, as activation area size increased, $\mathrm{KCN}$ misidentifications decreased and the performance even
TABLE I

Optimal ROC Analysis Results of KCN, CCA, and $t$ Test Under Different Activation Area Sizes. The First Number in Each Rectangle Is Sensitivity and the Second Is Specificity

\begin{tabular}{|c|c|c|c|c|c|c|}
\hline Method Area & $0.087 \%$ & $0.2 \%$ & $0.33 \%$ & $0.5 \%$ & $0.67 \%$ & $\mathbf{0 . 8 3} \%$ \\
\hline & 1 & 1 & 0.99 & 0.977941 & 1 & 1 \\
\hline $\mathrm{KCN}$ & 0.991049 & 0.999371 & 0.999489 & 0.999016 & 0.998934 & 0.999209 \\
\hline & 1 & 1 & 0.98 & 0.992647 & 1 & 1 \\
\hline $\mathrm{CCA}$ & 0.999136 & 0.991823 & 0.992992 & 0.993415 & 0.991981 & 0.997663 \\
\hline & 1 & 1 & 1 & 0.992647 & 0.994505 & 1 \\
\hline$t$-test & 0.999529 & 0.991352 & 0.999803 & 0.992903 & 0.997511 & 0.998099 \\
\hline
\end{tabular}

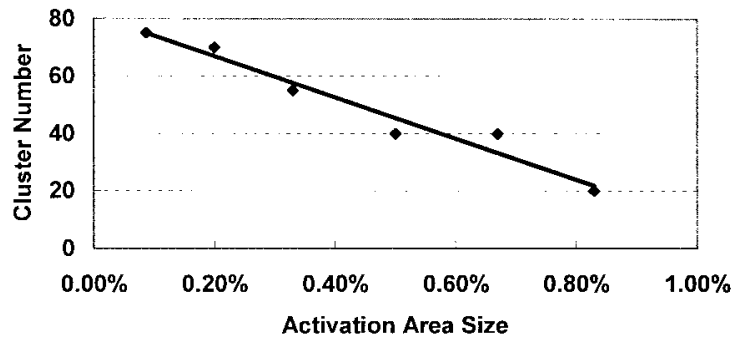

Fig. 5. The relation between the KCN cluster number with the optimal sensitivity and specificity and activation area size. Notice that the optimal cluster number decreased nearly linearly as area size increased.

surpassed both model-based methods when area size was larger than $0.2 \%$ of the head size.

Furthermore, two other important properties were observed. One was that when the activated area size decreased, the cluster number needed to reach optimal sensitivity and specificity increased nearly linearly (Fig 5). This suggested the use of more clusters when smaller activation regions were to be discriminated or when the size of input data was larger (e.g., more slices). The other was that when the activation area size was the same, specificity still increased monotonically along with cluster number, but sensitivity dropped dramatically when the cluster number exceeded a certain value due to over classification [Fig. 6(a)]. The use of FCM in the second stage could bring back these over classified patterns and elevate its sensitivity [Fig. 6(b)].

3) Effect of Phase Delay: Under the smallest phase lag condition (phase delay $=\pi / 6$ ) the performance of both modelbased methods dropped severely due to the bias of their false model (Fig. 7). KCN, on the other hand, was not affected by any phase delay as we had expected. For a larger phase lag, both CCA and $t$ test could not find any well matched waveforms and became nearly a random guess (Table II).

4) Effect of Complex Response: When the actual waveform contained adaptation-like and undershoot properties, both model-based methods, due to their false assumptions, tended to find the regions with responses acting as the boxcar paradigm. Thus, this made their averaged waveforms relatively flat during and after the stimulating state. Only KCN could identify the actual fatigue- or adaptation-like response and undershoot precisely. 


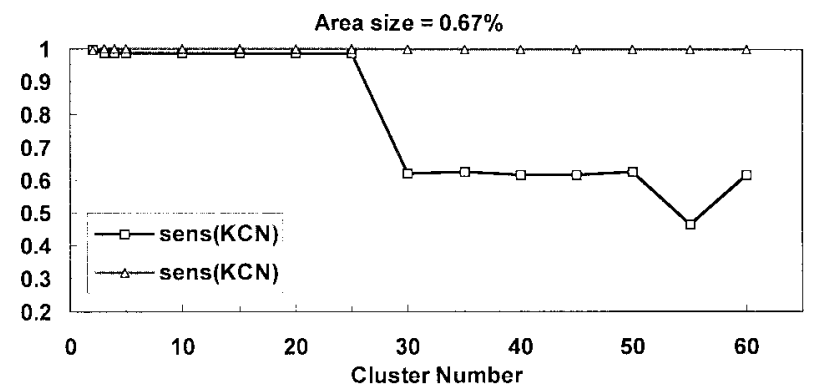

(a)

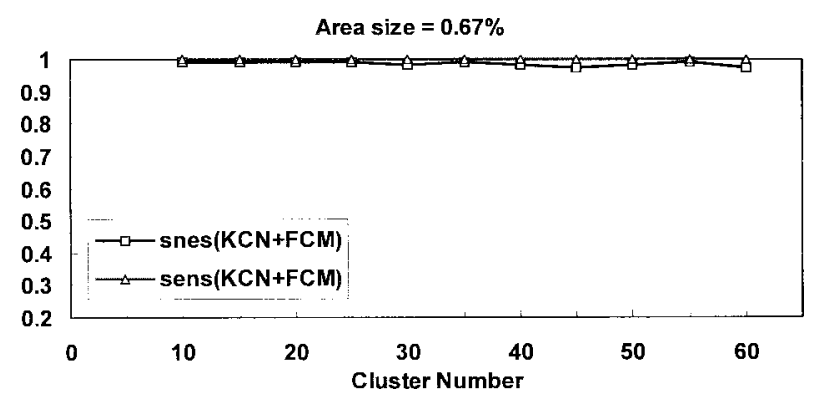

(b)

Fig. 6. The trends of sensitivity (the lines marked as sens) and specificity (marked as spec) with respect to the cluster number under the activation region occupying $0.67 \%$ of head area (a) before and (b) after using FCM to reorganize the features identified by $\mathrm{KCN}$. When we used $\mathrm{KCN}$ only, increasing the cluster number too much decreased sensitivity due to over classification of response features. After applying FCM, the over classified features were rejoined and, hence, raised the sensitivity.

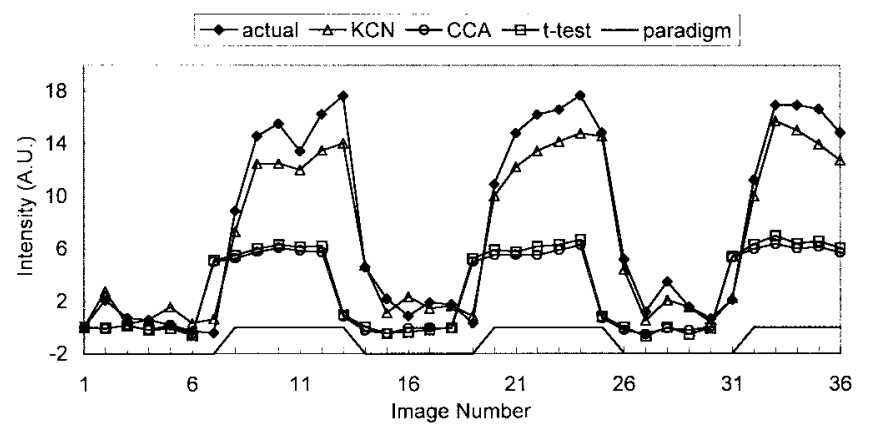

Fig. 7. When the phase delay was $\pi / 6$, the averaged waveforms in the activation regions identified by $\mathrm{CCA}$ and test were biased by the assumed paradigm. On the contrary, $\mathrm{KCN}$ found the delayed response exactly.

TABLE II

Optimal ROC Analysis Results of KCN, CCA, AND $t$ Test Under Different Phase Delays. The First Number in Each Rectangle Is Sensitivity and the Second Is Specificity

\begin{tabular}{c|c|c|r|c}
\hline Method Delay & 0 & $\pi / 6$ & $\pi / 3$ & $\pi / 2$ \\
\hline \multirow{2}{*}{ KCN } & 0.999371 & 0.993671 & 0.99226 & 0.994067 \\
& 1 & 0.916667 & 0.8 & 0.166667 \\
\hline \multirow{2}{*}{$\mathbf{C C A}$} & 0.991823 & 0.963755 & 0.811699 & 0.815591 \\
& 1 & 0.9 & 0.8 & 0.116667 \\
\hline \multirow{2}{*}{-test } & 0.991352 & 0.974094 & 0.841576 & 0.843266 \\
\hline
\end{tabular}
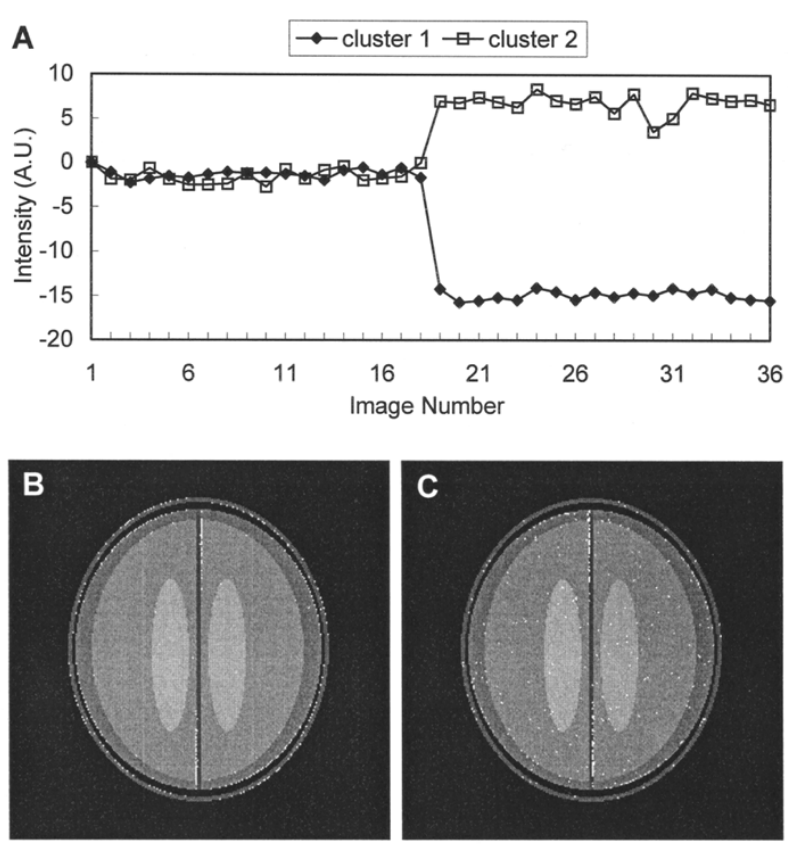

Fig. 8. (a) Two patterns related to a sudden head rotation of $0.2^{\circ}$ occurred at the 19th image. The patterns of clusters 1 and 2 represented the motion that occurred at (b) one side of edge and (c) the other side, respectively. This suggested that KCN could be used to check the presence of slight head motion.

TABLE III

Comparison of COMPUTATION Time EFFiCiency. KCN AND FCM REPRESENTS Using KCN AND FCM Alone to ANAlYZE. KCN + fCM Cascades Both Methods As Described in Section II

\begin{tabular}{c|r|r|r|r|r|r|r|r|r}
\hline $\begin{array}{c}\text { Method } \\
\text { Clusier Number }\end{array}$ & \multicolumn{1}{|c|}{$\mathbf{5}$} & $\mathbf{1 0}$ & $\mathbf{1 5}$ & $\mathbf{2 0}$ & $\mathbf{2 5}$ & $\mathbf{3 0}$ & $\mathbf{3 5}$ & $\mathbf{4 0}$ & $\mathbf{4 5}$ \\
\hline KCM & 9180 & 20787 & $\mathbf{3 2 9 1 1}$ & $\mathbf{4 7 6 3 8}$ & 61191 & $\mathrm{~N} / \mathrm{A}$ & $\mathrm{N} / \mathrm{A}$ & $\mathrm{N} / \mathrm{A}$ & $\mathrm{N} / \mathrm{A}$ \\
\hline KCN & 38 & 38 & 125 & 190 & 278 & 496 & 559 & 796 & 837 \\
\hline
\end{tabular}

5) Effect of Head Motion: For a rotation of less than $0.2^{\circ}$, no motion could be observed when displayed in the CINE mode and no motion-related pattern was identified by $\mathrm{KCN}$. When the rotation equaled $0.2^{\circ}$, two patterns related to head motion were identified (Fig. 8). Both patterns showed that a step at the time rotation occurred and their associated regions were located at the edges. This suggested that this analysis method could be used to find minor head motion.

6) Computational Time Efficiency: As we had expected, CCA and $t$ test were extremely fast-both took just a few seconds in our test. For KCN and FCM the processing time was much longer and was proportional to the cluster number (Table III). Comparing both methods, FCM was far slower than $\mathrm{KCN}$, thus, choosing $\mathrm{KCN}$ as the first-stage classifier was much more efficient. Although FCM was less efficient, cascading it in the second stage merely increased processing time a little for it only needed to process the features identified by $\mathrm{KCN}$. 


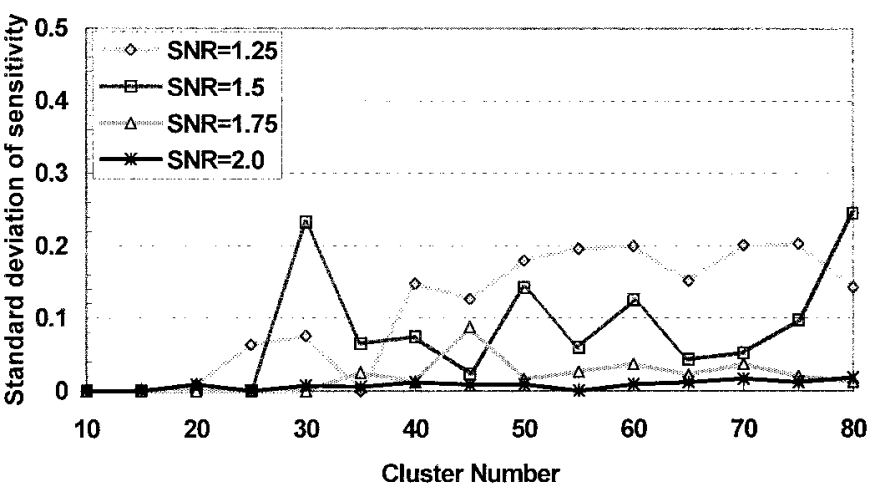

Fig. 9. Standard deviation of sensitivity of $\mathrm{KCN}$ using different initial values versus cluster number under $\Delta \mathrm{SNR}=1.25,1.5,1.75$, and 2.0. The sensitivity to initial values enlarged as noise level increased. This variation also increased with cluster number due to the increasing complexity of the local minimum. For $\Delta \mathrm{SNR} \geq 1.75, \mathrm{KCN}$ performed very stably.

7) Sensitivity to Initial Values: Generally, the variation (measured by the standard deviation) of sensitivity under different initial values increased with the noise level. When $\Delta$ SNR was higher than $1.75, \mathrm{KCN}$ performed very stably (Fig. 9). In addition, the variation in sensitivity also tended to increase with the cluster number (although with one or two zigzags). This may be due to the increasing complexity of the possible solutions to the optimization problem. Therefore, it was easier to converge to other local minimums and it increased the variability.

\section{B. Human Studies}

1) Regular Versus Arbitrary Paradigm: For the regular paradigm experiment, analysis results of one subject were shown in Fig. 10. Two out of seven patterns identified by our proposed method corresponded to the paradigm. $C C$ of these patterns with respect to the paradigm all exceeded 0.7 . Comparing the activation regions of these clusters with that of CCA $(p<0.001$, corresponding $C C>0.358)$, and $t$ test $(p<0.001$, two tailed, corresponding $t$-value $>3.21)$ results, all methods identified the activation site on the primary motor cortex of the left hemisphere successfully. However, CCA and $t$ test misidentified many unrelated regions as active, especially those on the opposite hemisphere.

One important thing worth noting was that after inspecting the activation regions found by CCA or $t$ test more closely, the large activation region on the left hemisphere actually contained at least two kinds of signal sources. One was from the motor cortex (the actual functioning site) and the other from the outside region of the motor cortex (a possible location of cortical venous vessels). While both model-based methods could not tell these different kinds of signals from each other, our proposed method discriminated them successfully and found that the signal change from the cortical region was much smaller than that from the cortical veins [Fig. 10(e)].

For the arbitrary paradigm experiment, both CCA and $t$ test could not be used due to the lack of exact information about the paradigm. On the contrary, our proposed method showed great success in this kind of experiment. After they were classified into 30 clusters by $\mathrm{KCN}$ and then into seven
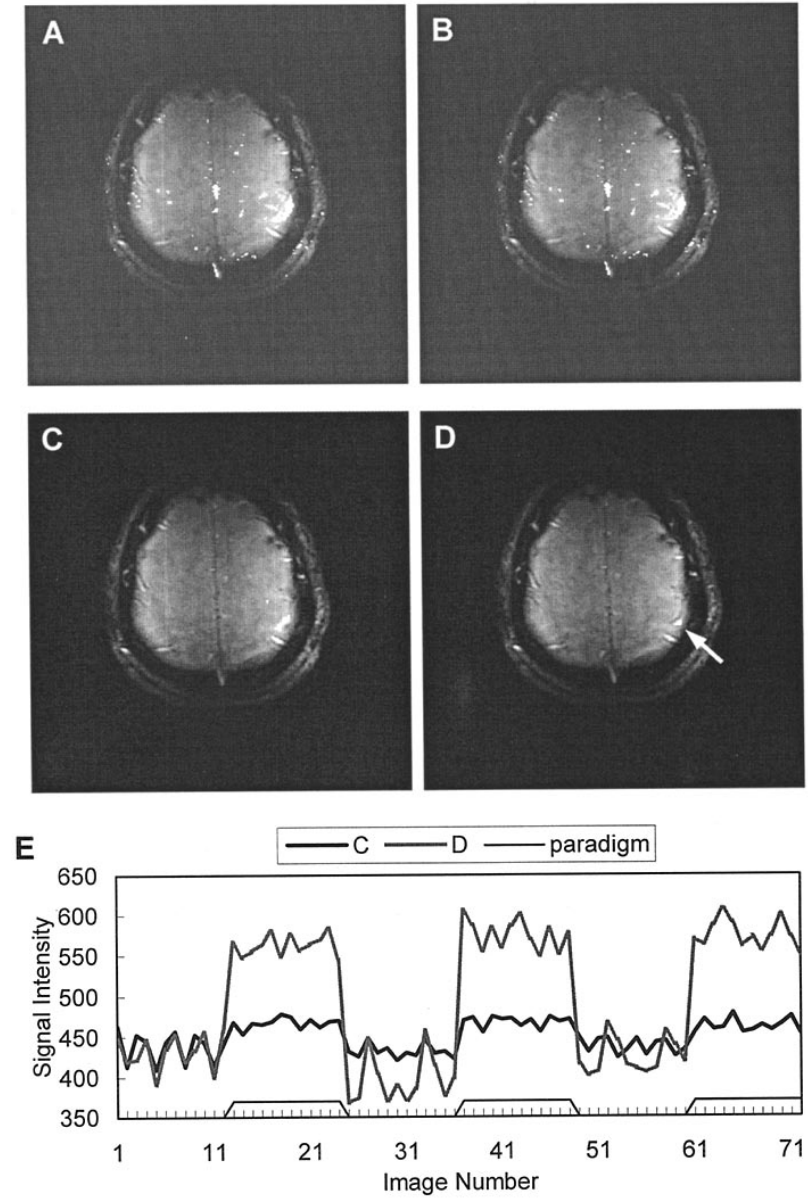

Fig. 10. Activation maps formed by (a) CCA $(p<0.001)$ and by (b) $t$-test $(p<0.001$, two tailed) overlaid on the first image of the fMRI sequence in the regular paradigm experiment. Both maps revealed the activation on the motor cortex of the left hemisphere, but they also misidentified many unrelated regions as active, especially those on the opposite hemisphere. Activation regions found by our proposed method (c) and (d) also showed the activation on the left motor cortex, but without any misidentification on the opposite part. (e) Both features correlated very well with the paradigm-their $C C$ were 0.889 and 0.8932 , respectively. The signal change in (d) (signal change $=27.4 \%$ ), which may come from larger venous vessels, was much larger than that in $(\mathrm{c})$ ( signal change $=6.95 \%$ ).

clusters by FCM, responses of three of the clusters seemed to represent some kind of functional activity patterns and their associated regions were all around the motor cortex (Fig. 11). Compared with the actual paradigm determined afterwards by the MRI operator, the responses of these clusters were exactly coincident with the actual paradigm [Fig. 11(d)]. All of their $C C$ with respect to the actual paradigm were higher than 0.75 . Therefore, by merely looking at these regions and patterns, one could determine from the anatomy what kind of activation task it was and determine its exact activation pattern using this technique.

2) Blind Test: Data was first classified into 50 clusters by $\mathrm{KCN}$, and then into fewer clusters by FCM. One pattern was found to contain the functional activation pattern (Fig. 12). Its activation region and pattern both resembled the map and averaged waveform obtained using CCA $(C C>0.5)$ with the actual paradigm transmitted from KVGH after this blind test. The subject actually performed a signature-writing task 

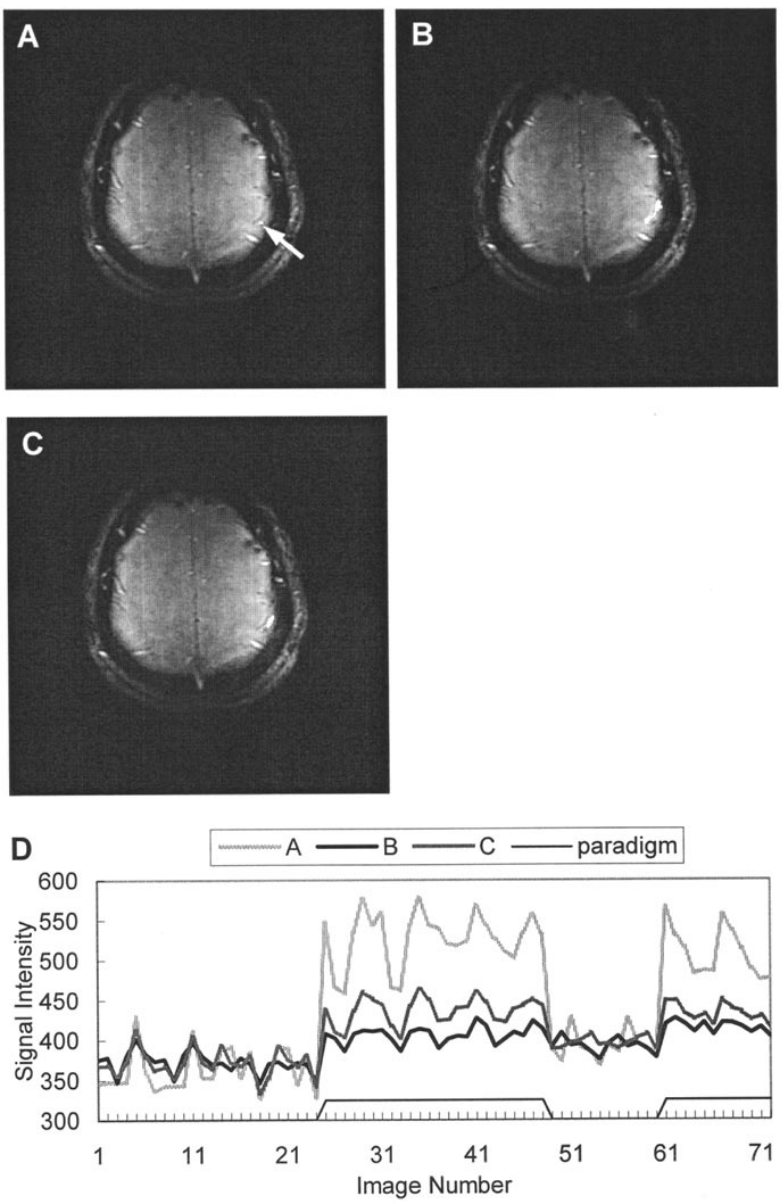

Fig. 11. Activation regions of three clusters, (a), (b), and (c), respectively, identified by our proposed method in the arbitrary paradigm experiment. (d) The patterns of these clusters not only correlated very well with respect to the actual paradigm ( $C C$ were $0.8826,0.7576$ and 0.8179 , respectively), their associated regions were all around the motor cortex.

using his right hand. In addition to obtaining nearly the same result as CCA, our proposed method could identify three subclusters in this activation-related region (Fig. 13). Two were on the primary motor cortex, but with different signal change percentages. The other was on the premotor area and with larger signal variation, perhaps due to the complex hand movement associated with signature writing.

\section{DISCUSSIONS}

\section{A. Effect of Noise}

From phantom studies, it was shown that the major limitation of this analysis method was noise level. Model-based methods, with the help of the functional response model, can achieve acceptable results, even when $\Delta$ SNR was as low as one. Since a high noise level made the feature of functional response less clear, $\mathrm{KCN}$ could not converge to its optimum point easily, even when using many clusters. Although this method suffered from high noise level, let us not forget the goal of fMRI is not only to find the known response but also the unknown activation pattern and the associated region through the analysis method. This is what this method focuses on. Even if CCA and $t$ test were less vulnerable to noise, their
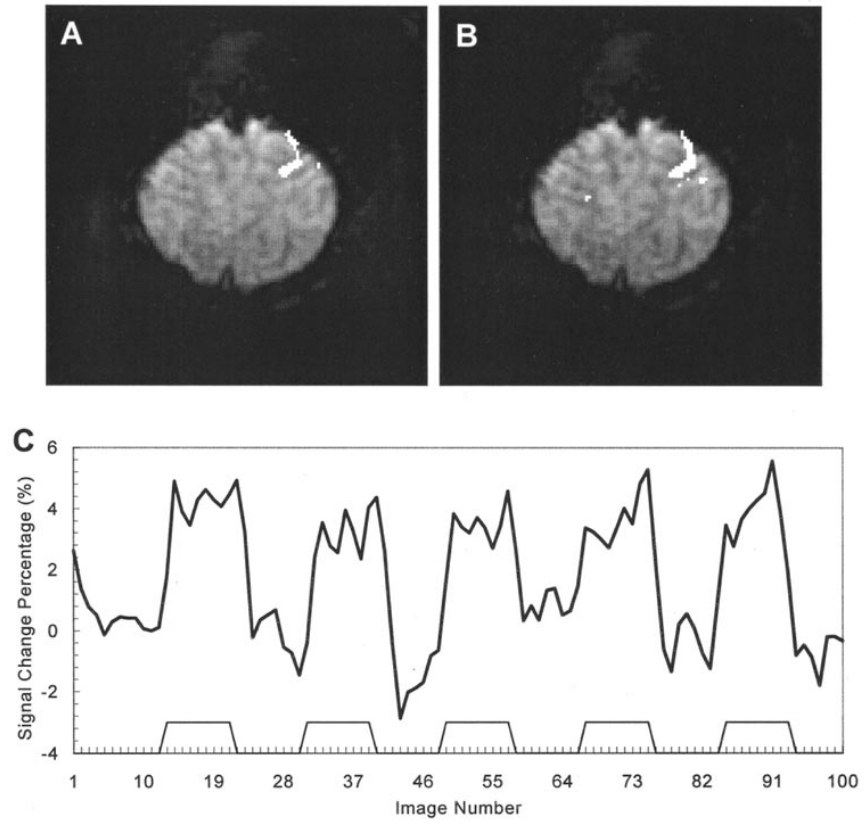

Fig. 12. (a) Activation map formed by clustering original images into 50 clusters by $\mathrm{KCN}$ and then reorgnized into fewer clusters by FCM in blind test study. (b) Correlation coefficient map $(\mathrm{CC}>0.5)$ overlaid on the first image of the fMRI series. It can be noted that CCA misidentified a small region on the opposite hemisphere while our cascade method did not. (c) The identified pattern correlated very well with the actual paradigm supplied by remote MR site after this blind test.

identified response patterns and activation regions are biased by the assumed response and will certainly lead to inaccurate results when the actual response waveform does not resemble the estimated model. This is one of the important issues in analyzing and interpreting single-trial fMRI results [35].

To overcome the low sensitivity under the noisy situation we tested using a Gaussian filter in the spatial domain. With a $7 \times 7$ (pixels) window size and full-width at half-maximum (FWHM) of 2 pixels, sensitivity could be elevated to near one with very few misidentifications and with little blurring of the activation region (Fig. 14). In fact, in order to gain the benefit of high sensitivity while eliminating the disadvantage of the assumed response model in CCA, one could apply this modelfree method to get the actual response and take this waveform as a reference for the further analysis of CCA or $t$ test.

\section{B. Influence of Activation Area Size}

When a certain feature occurred more frequently in the input data, the more the output cluster related to this feature was trained. Thus, this feature could be identified more easily and more precisely, even when using fewer clusters. In other words, under the same noise level, a pattern of smaller area size would be influenced by other patterns, making it harder to identifiy. If one wanted to find an activation pattern of small area size, he could use more clusters or apply spatial filtering to elevate sensitivity and specificity. However, if the cluster number exceeded a certain value, $\mathrm{KCN}$ could over classify the pattern of the larger area and make the pattern split into two or more subpatterns as well as two or more subactivation regions, making the sensitivity decrease dramatically. This 

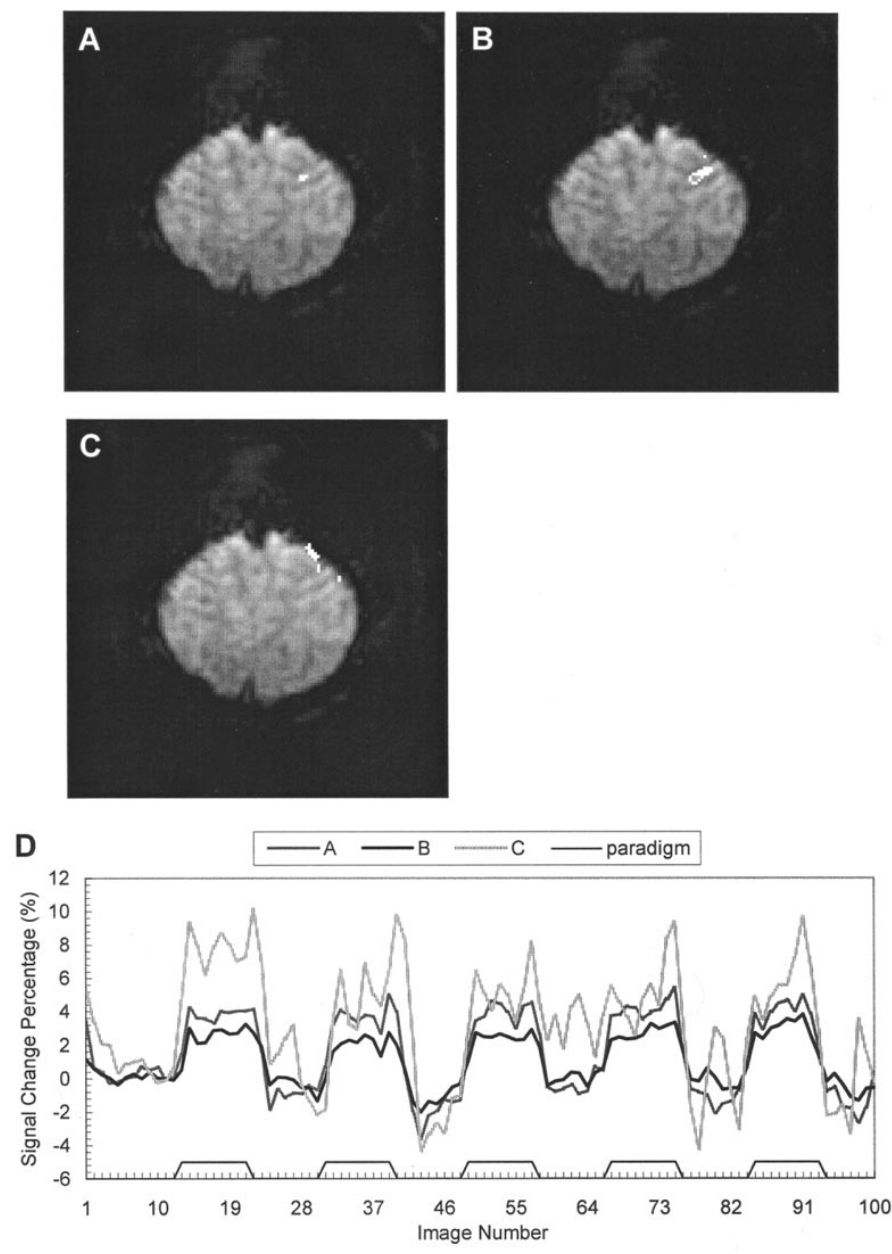

Fig. 13. The activation region identified by our cascade method in Fig. 10 actually contained three different $\mathrm{KCN}$ patterns. Two were from the primary motor area [(a) and (b)], and the other was from premotor area (c). (d) From the averaged waveforms in these clusters it could be seen that (a) and (b) had similar temporal variation but signal changes in (a) $(4.11 \%)$ and (c) $(4.24 \%)$ were both larger than that in (b) $(2.51 \%)$.

problem was overcome by incorporating FCM to merge the over classified features.

\section{How Many Clusters Are Sufficient?}

The complexity of physiological activities and other artifacts during a functional experiment make it difficult to determine the optimal cluster number in advance. The noise level of the acquired images and the activation area size of interest also affect the selection of the cluster number. Increasing the cluster number could elevate specificity and make the pattern of the smaller area size easier to identify. When noise level is low, a lower cluster number is needed. However, too many clusters will increase processing time and make the result hard to analyze. There should be a tradeoff between effectiveness and efficiency. Usually we choose 30 to 50 clusters for $\mathrm{KCN}$ and 5 to 10 clusters for FCM for FLASH fMRI studies. If the activated region being investigated is very small compared with the brain area, more clusters are desirable.

One thing to be noted here is that although we treated the cluster number as the threshold value in ROC analysis, it is
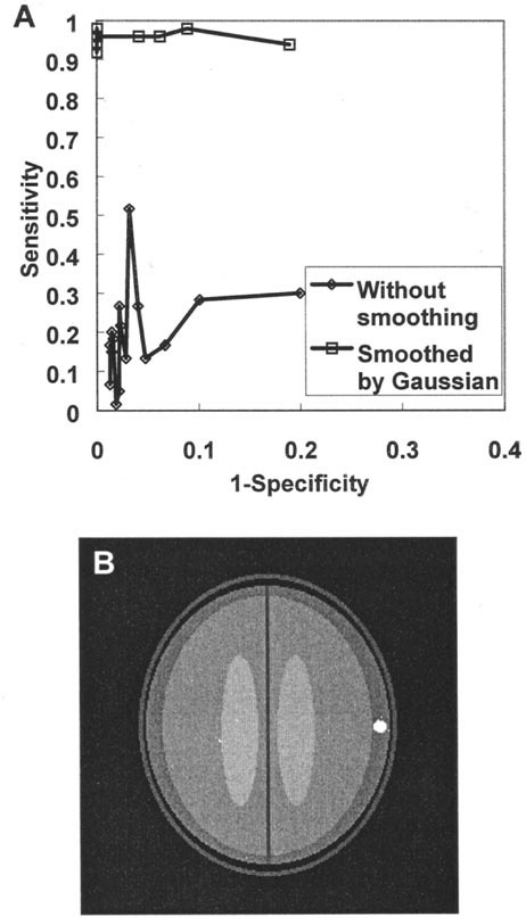

Fig. 14. (a) ROC curve of $\mathrm{KCN}$ under the noisiest condition $(\Delta \mathrm{SNR}=$ 1.0) was elevated to close to the perfect situation after images were smoothed by a Gaussian filter with FWHM $=2$ pixels. (b) There were very few misidentifications and little blurring of the activation region after smoothing.

actually not equivalent to a threshold in ROC. Increasing this threshold did increase specificity, but sensitivity did not show an apparent decreasing trend. Under low noise level or a large activation area condition, sensitivity only fluctuated slightly at a certain level until over classification occurred. When a signal became noisier, the variation in sensitivity enlarged and tended to vary randomly. This meant that increasing the cluster number, unlike increasing the threshold, did not necessarily decrease the sensitivity. Therefore, one should not regard cluster number as a threshold in determining the optimal cluster number, especially when the noise level was high or the activation area was small.

There are several possible ways to solve the problem of the unknown optimal cluster number. One is using a cluster discovery network, such as adaptive resonance theory 1 (ART 1) [39], to add cluster automatically if some pattern is quite different from existing cluster features. Using parameter estimation techniques in image classification or segmentation to estimate the optimal cluster number is another approach [40].

\section{Computation Time Efficiency}

Although our method was more efficient than the FCMbased clustering method, it still required tens of minutes of computation time to process usual multislice echo-planar imaging (EPI) fMRI data. To further shorten the processing time there are two possible approaches. One is to reduce the cluster number by merging redundant clusters during the learning process, as proposed by Jarmasz et al. [28]. The other is to decrease the size of the input data. Since most brain 
regions contained no stimulus-related activity and the variation of the time-course signal in these regions was usually small, we could eliminate clustering these regions by setting a threshold on temporal variation.

\section{E. Influence of Initial Values}

Although $\mathrm{KCN}$ is less sensitive to initial values, improper initial values still affect the analysis ability of this method, especially under noisy condition. FCM also suffers from this problem. Improper initial values would make both clustering processes hard to converge, taking longer processing time and converging to the local minimum, thus producing unsatisfactory results. Since we still lack the best criterion for initialization, rerunning the clustering procedure seems to be the only solution when the analysis result is not very good. In our studies, we found that initializing $\mathrm{KCN}$ with zero vectors was a proper alternative.

\section{F. Ability to Discriminate Large Venous Vessels or Other Signal Sources}

Response from a larger activation area could be identified easier even under lower $\Delta$ SNR. In other words, a lower level of activation with a larger area or a higher level of activation with a smaller area could be identified more accurately. This could explain why the signal from larger draining veins, which has a larger signal change due to flow-related enhancement [36], [37], could be separated from the BOLD signal from the cortical region in our study using a conventional gradient echo sequence. Even if images were acquired by ultra fast pulse sequences, such as EPI or spiral, locations of larger draining veins could also be identified, for they have longer delay time than signal from gray matter [38]. So this method could be helpful in separating the contribution from larger venous vessels and could provide a finer definition of the actual activation region. In addition, since the temporal properties of physiological motion, such as respiration, induced signal changes are different from stimulus-related activity, these signals could be extracted using this model-free analysis method.

\section{G. Quantitative fMRI}

Since current fMRI methodology lacks quantitative analysis abilities, how to extract quantitative parameters that can be used to describe neuronal function is one of the most important topics in fMRI studies. Some of the parameters of fMRI results seem to relate to different levels of neuronal activities. For instance, according to Price et al. [41], the activated area size may be linked to the recruitment of active neurons under a motor experiment with different complexity. Since this model-free analysis method could provide much more precise activation maps of different activation response patterns, counting and comparing the activation area size became very easy. In addition, temporal characteristics of functional activities may also have a connection with quantitative studies such as calculating delay time and amplitude of the peak response. The identified feature waveform produced by this method would also be useful in examining these properties.
Thus, this method would be very helpful in studying and comparing not only qualitative but also quantitative fMRI studies.

\section{CONCLUSION}

The analysis power and properties of an efficient model-free analysis method for fMRI has been demonstrated. Compared with current clustering methods, this method can efficiently and stably extract activities with different signal change levels, phase delays, etc., from very small regions under moderate noise level and proper selection of the cluster number. Moreover, activities coming from different signal sources or other noise sources such as head motion can be identified. Thus, this method can provide a finer definition of the actual activation region and a new window to inspect the actual physiological activities in the working brain. The blind studies also show the potential of this method to find voluntary activation patterns and cerebral areas with fMRI alone. Further efforts will be focused on improving the processing time, determining the optimal cluster number, and statistical interpretation of identified patterns. Quantitative fMRI is also being studied at this moment.

\section{ACKNOWLEDGMENT}

The authors would like to thank Dr. K.-M. Huang and P.-J. Chiang of National Taiwan University Hospital for conducting the experiment. We also thank Dr. M.-T. Wu of Kaohsiung Veterans General Hospital for providing blind test data and helpful discussion.

\section{REFERENCES}

[1] K. K. Kwong, J. W. Belliveau, D. A. Chesler, I. E. Goldberg, R. M Weisskoff, B. P. Poncelet, D. N. Kennedy, B. E. Hoppel, M. S. Cohen, R. Turner, H.-M. Chen, T. J. Brady, and B. R. Rosen, "Dynamic magnetic resonance imaging of human brain activity during primary sensory stimulation," Proc. Natl. Acad. Sci. USA, vol. 89, pp. 5675-5679, 1992.

[2] S. Ogawa, D. W. Tank, R. Menon, J. M. Ellermann, S.-G. Kim, H. Merkle, and K. Ugurbil, "Intrinsic signal changes accompanying sensory stimulation: Functional brain mapping with magnetic resonance imaging," Proc. Natl. Acad. Sci. USA, vol. 89, pp. 5951-5955, 1992.

[3] P. A. Bandettini, E. C. Wong, R. S. Hinks, R. S. Tikofsky, and J. S Hyde, "Time course EPI of human brain function during task activation," Magn. Reson. Med., vol. 25, pp. 390-397, 1992.

[4] J. Frahm, K. Merboldt, and W. Hanicke, "Functional MRI of human brain activation at high spatial resolution," Magn. Reson. Med., vol. 29, pp. 139-144, 1992.

[5] K. K. Kwong, "Functional magnetic resonance imaging with echo planar imaging," Magn. Reson. Q., vol. 11, no. 1, pp. 1-20.

[6] J. L. Boxerman, P. A. Bandettini, K. K. Kwong, J. R. Baker, T. L. Davis, B. R. Rosen, and R. M. Weisskoff, "The intravascular contribution to fMRI signal change: Monte Carlo modeling and diffusion-weighted studies in vivo," Magn. Reson. Med., vol. 34, pp. 4-10, 1995.

[7] S. Ogawa, T. M. Lee, and B. Barrere, "The sensitivity of magnetic resonance image signals of a rat brain to changes in the cerebral venous blood oxygenation," Magn. Reson. Med., vol. 29, pp. 205-210, 1993.

[8] P. A. Bandettini, A. Jesmanowicz, E. C. Wong, and J. S. Hyde, "Processing strategies for time-course data sets in functional MRI of the human brain," Magn. Reson. Med., vol. 30, pp. 161-173, 1993.

[9] K. J. Friston, P. Jezzard, and R. Turner, "Analysis of functional MRI time-series," Human Brain Mapping, vol. 1, pp. 153-171, 1994.

[10] R. T. Constable, G. McCarthy, T. Allison, A. W. Anderson, and J. C. Gore, "Functional brain imaging at $1.5 \mathrm{~T}$ using conventional gradient echo MR imaging technique," Magn. Reson. Imag., vol. 11, pp. 451-459, 1993. 
[11] J. R. Baker, R. M. Weisskoff, and C. E. Stern et al., "Statistical assessment of functional MRI signal change," in Proc. SMR 2nd Annu. Meeting, San Francisco, CA, 1994.

[12] S. Kollias, X. Golay, and D. Meier et al., "Blood oxygenation level dependent (BOLD) signal response to progressive shortening of the rest period between constant activated phases at high temporal resolution," in Proc. ISMRM 4th Annu. Meeting, New York, NY, 1996. p. 1758 .

[13] G. Kruger, J. Frahm, and A. Kleinschmidt, "The cerebral blood oxygenation response to functional challenge: Differences between human motor and visual cortex," in Proc. ISMRM 4th Annu. Meeting, New York, NY, 1996, p. 1757.

[14] R. L. Buckner, P. A. Bandettini, K. M. O’Craven, R. L. Savoy, S. E. Petersen, M. E. Raichle, and B. R. Rosen, "Detection of corteical activation during averaged single trials of a cognitive task using functional magnetic resonance imaging," Proc. Natl. Acad. Sci. USA, vol. 93, pp. 14878-14883, 1996.

[15] X. Hu, T. H. Le, and K. Ugurbil, "Evaluation of the early response in fMRI in individual subjects using short stimulus duration," Magn. Reson. Med., vol. 37, pp. 877-884, 1997.

[16] G. M. Boynton, S. A. Engel, G. H. Glover, and D. J. Heeger, "Linear systems analysis of functional magnetic resonance imaging in human V1," J. Neurosci., vol. 16, pp. 4207-4221, 1996.

[17] J. J. Sychra, P. A. Bandettini, N. Bhattacharya, and Q. Lin, "Synthetic images by subspace transforms I. Principal components images and related filters," Med. Phys., vol. 21, pp. 193-201, 1994

[18] W. Backfrieder, R. Baumgartner, M. Samal, E. Moser and H. Bergmann, "Quantification of intensity variations in functional MR images using Rotated principal components," Phys. Med. Biol., vol. 41, pp. 1425-1438, 1996

[19] M. J. McKeown, T.-P. Jung, S. Makeig, G. G. Brown, S. S. Kindermann, T.-W. Lee, and T. J. Sejnowski, "Spatially independent activity patterns in functional magnetic resonance imaging data during the stroop colornaming task," Proc. Natl. Acad. Sci. USA, vol. 95, pp. 803-810, 1998.

[20] M. J. McKeown, S. Makeig, G. G. Brown, T.-P. Jung, S. S. Kindermann, A. J. Bell, and T. J. Sejnowski, "Analysis of fMRI data by blind separation into independent spatial components," Human Brain Mapping, vol. 6, no. 3, pp. 160-188, 1998.

[21] G. Scarth, M. McIntrye, B. Wowk, and R. L. Somorjai, "Detection novelty in functional images using fuzzy clustering," in Proc. SMR $3 \mathrm{rd}$ Annu. Meeting, Nice, France, 1995, p. 238.

[22] H. Fischer and J. Hennig, "Clustering of functional MR data," in Proc. ISMRM 4th Аnпu. Meeting, New York, NY, 1996, p. 1779.

[23] J. C. Bezdek, Pattern Recognition with Fuzzy Objective Function Algorithms. NY: Plenum, 1981

[24] G. Scarth, M. Alexander, M. McIntyre, B. Wowk, and R. L. Somorjai, "Artifact detection in fMRI using fuzzy clustering," in Proc. ISMRM 4th Annu. Meeting, New York, NY, 1996, p. 1783.

[25] R. Baumgertner, G. Scarth, C. Teichtmeister, R. Somorjai and E. Moser, "Fuzzy clustering improves reproducibility of gradient echo functional MRI by separating various contributions from venous vessels in the human visual cortex," in Proc. ISMRM 5th Annu. Meeting, Vancouver, BC, Canada, 1997, p. 1662.

[26] R. Baumgertner, C. Windischberger, and E. Moser, "Quantification in functional magnetic resonance imaging: Fuzzy clustering vs. correlation analysis," Magn. Reson. Imag., vol. 16, no. 2, pp. 115-125, 1998.

[27] X. Golay, S. Kollias, G. Stoll, D. Meier, A. Valavanis, and P. Boesiger "A new correlation-based fuzzy logic clustering algorithm for fMRI," Magn. Reson. Med., vol. 40, pp. 249-260, 1998.

[28] M. Jarmasz and R. L. Somorjai, "Time to join! Cluster-merging in unsupervised fuzzy clustering of functional MRI data," in Proc. ISMRM 6th Аnпи. Meeting, Sydney, Australia, 1998, p. 2068.

[29] K. H. Chuang, M. J. Chiu, C. C. Lin, and J. H. Chen, "Finding different physiological signal sources of fMRI data using paradigm-free Kohonen clustering network and fuzzy c-means," in Proc. ESMRMB 15th Annu. Meeting, Geneva, Switzerland, 1998, pp. 143-144.

[30] T. Kohonen, Self-Organizing Maps. NY: Springer-Verlag, 1995.

[31] N. R. Pal, J. C. Bezdek, and E. C.-K. Tsao, "Generalized clustering networks and Kohonen's self-organizing scheme," IEEE Trans. Neural Networks, vol. 4, pp. 549-556, July 1993.

[32] R. T. Constable, P. Skudlarski, and J. C. Gore, "An ROC approach for evaluating functional brain MR imaging and postprocessing protocols," Magn. Reson. Med., vol. 34, pp. 57-64, 1995.

[33] J. A. Sorenson and X. Wang, "ROC methods for evaluation of fMRI techniques," Magn. Reson. Med., vol. 36, pp. 737-744, 1996.

[34] J. V. Hajnal, R. Myers, A. Oatridge, J. E. Schwieso, I. R. Young, and G. M. Bydder, "Artifacts due to stimulus correlated motion in functional imaging of the brain," Magn. Reson. Med., vol. 31, pp. 283-291, 1994.

[35] J. Chen, E. Yacoub, and X. Hu, "Detection of the negative early response in fMRI using model-free neural networks analysis," in Proc. ISMRM 6th Annu. Meeting, Sydney, Australia, 1998, p. 251.

[36] J. Frahm, K.-D. Merboldt, W. Hanicke, A. Kleinschmidt, and H Boecker, "Brain or vein-oxygenation or flow? On signal physiology in functional MRI of human brain activation," NMR Biomed., vol. 7 , pp. 45-53, 1994.

[37] J. H. Duyn, C. T. W. Moonen, G. H. van Yperen, R. W. de Boer, and P R. Luyten, "Inflow versus deoxyhemoglobin effects in BOLD functional MRI using gradient echoes at 1.5T," NMR Biomed., vol. 7, pp. 83-88, 1994.

[38] A. T. Lee, G. H. Glover, and C. H. Meyer, "Discrimination of large venous vessels in time-course spiral blood-oxygen-level-dependent magnetic resonance functional neuroimaging," Magn. Reson. Med., vol. 33, pp. 745-754, 1995

[39] G. A. Carpenter and S. Grossberg, "A massive parallel architecture for a self-organizing neural pattern recognition machine," Comput. Vision, Graph., Image Processing, vol. 37, pp. 54-115, 1987.

[40] Z. Liang, R. J. Jaszczak, and R. E. Coleman, "Parameter estimation of finite mixtures using the EM algorithm and information criteria with application to medical image processing," IEEE Trans. Nucl. Sci., vol. 39, pp. 1126-1133, 1992.

[41] R. R. Price, H. Lee, and L. Welch et al., "Functional MR imaging assessment of fine motor function," in Proc. ISMRM 4th Annu. Meeting, New York, NY, 1996, p. 1763. 